Michael Eller, MD

Amy A. Gelfand, MD

Nina Y. Riggins, MD

Stephen Shiboski, PhD

Christoph Schankin, MD

Peter J. Goadsby, MD, $\mathrm{PhD}$

Correspondence to Dr. Goadsby: peter.goadsby@kcl.ac.uk

\section{Exacerbation of headache during dihydroergotamine for chronic migraine does not alter outcome}

\section{ABSTRACT}

Objective: To evaluate whether headache exacerbation associated with IV dihydroergotamine (DHE) infusion predicts medium-term headache outcome in patients with chronic migraine.

Methods: This was a retrospective chart review study of the UCSF Headache Center's use of IV DHE for chronic migraine from 2008 to 2012 . Medium-term headache outcome was assessed at 6-week follow-up. Univariate and multivariate logistic regression models were used to assess for predictors of outcome.

Results: Patients with chronic migraine $(n=274)$ were treated with a course of IV DHE. Of 214 with 6-week follow-up, 78\% had medium-term headache benefit. In a univariate logistic regression model, headache exacerbation with DHE was associated with lower odds of a positive medium-term headache outcome (odds ratio [OR] 0.43, 95\% confidence interval [CI] 0.200.91). However, in the multivariate logistic regression model, headache exacerbation was no longer an independent predictor of treatment outcome (OR 0.65, 95\% Cl 0.28-1.51). Factors that independently predicted outcome were nausea (OR 0.12, 95\% Cl 0.02-1.00, p = 0.05), age (OR 1.68 for each decade increase in age, $95 \% \mathrm{Cl} 1.24-2.28)$, and medication overuse (OR 0.42, 95\% Cl 0.18-0.97).

Conclusions: After controlling for nausea and other factors, headache exacerbation with DHE infusions is not an independent predictor of poor headache outcome and clinicians should not interpret its presence as a reason to stop treatment. The focus of management should be on controlling nausea as it is the most important modifiable factor in achieving a good headache outcome with an inpatient course of IV DHE for chronic migraine. Neurology ${ }^{\circledR}$ 2016;86:856-859

\section{GLOSSARY}

$\mathbf{C I}=$ confidence interval; $\mathbf{C M}=$ chronic migraine; $\mathbf{D H E}=$ dihydroergotamine; ICHD-3 beta = International Classification of Headache Disorders, 3rd edition (beta version); OR = odds ratio; UCSF = University of California, San Francisco.

Chronic migraine (CM) affects approximately $2 \%$ of adults, and can be highly disabling. ${ }^{1} \mathrm{We}$ previously reported our development of a 5-day inpatient course of IV dihydroergotamine (DHE) to treat chronic migraine, which built on the initial 3-day course of Raskin. ${ }^{2}$ We reported $75 \%$ experiencing medium-term headache benefit and the importance of good nausea control. ${ }^{3}$

We have observed that some CM patients experience transient headache worsening during DHE infusions, which may be interpreted as reason to cease the infusions and thus contribute to poorer outcomes for patients. We undertook this chart review study to assess whether transient headache worsening with DHE predicts medium-term headache outcome in patients with CM.

METHODS Standard protocol approvals, registrations, and patient consents. This study was approved by the University of California, San Francisco (UCSF) Committee for Human Research (protocol numbers 12-09823 and 10-00020) under a minimal risk protocol.

Study design and study population. All CM patients admitted by the UCSF Headache Service between January 1, 2008, and December 31, 2012, for IV DHE were included. DHE therapy followed a standard, previously published protocol. ${ }^{3}$ Briefly, the protocol involves DHE administration in $1 \mathrm{mg}$ doses, after initial doses at 0.5 and $0.75 \mathrm{mg}$, over 5 days to a typical target dose of

From the Headache Group, Department of Neurology (M.E., A.A.G., N.Y.R., C.S., P.J.G.), and Epidemiology and Biostatistics (S.S.), University of California, San Francisco; and Headache Group, Basic \& Clinical Neuroscience, and NIHR-Wellcome Trust Clinical Research Facility (P.J.G.), King's College London, UK.

Go to Neurology.org for full disclosures. Funding information and disclosures deemed relevant by the authors, if any, are provided at the end of the article. 
$11.25 \mathrm{mg}$ with premedication using ondansetron or domperidone, or both. In patients with comorbid medication overuse (30\%) they also had IV aspirin ${ }^{4}$ prior to DHE. We retrospectively analyzed the prospectively collected inpatient headache diaries and contemporaneous progress notes and discharge summaries. Patients were not routinely asked if headache was exacerbated with DHE infusions, though they did keep headache diaries during the admission. We assessed a DHE worsening as headache, typically an exacerbation of the underlying phenotype, or nausea increase during or within 30 minutes of the infusion. Follow-up occurred outpatient approximately 6 weeks after discharge, at which time medium-term headache outcome was assessed by the treating clinician and recorded in the chart. Patients with opioid, barbiturate, triptan, or combination medication overuse were included.

Diagnoses of CM and medication overuse followed International Classification of Headache Disorders, 3rd edition, beta version (ICHD-III beta) criteria using documentation in the notes. All patients were seen by one of us (P.J.G.).

Data analysis. The primary predictor variable was headache exacerbation with DHE infusions. While the specific dose number associated with transient headache exacerbation was not always recorded, in almost all cases it was one of the first 3 doses given. The primary outcome variable was medium-term headache benefit (any degree of benefit) at 6-week follow-up as reported by the patient in answer to the following question: Did you derive any benefit in terms of your headache from the treatment? The marginal association between headache exacerbation with DHE infusions and medium-term headache benefit, as well as associations between other predictor variables and mediumterm headache benefit, were initially assessed using frequency table methods ( $\chi^{2}$ and Fisher exact, as appropriate) and univariate logistic regression models. Guided by the results of the marginal analyses, additional logistic models were fitted to account for the effects of potential confounding and mediating variables. To assess the potential role of nausea as a mediating influence on the effect of DHE therapy, separate models were fitted treating the former as a predictor of the latter, and including both jointly in a model for the outcome. This model also adjusted for medication overuse, leg cramping as marker of DHE binding to $5 \mathrm{HT}_{1 \mathrm{~B}}$ receptors in the saphenous vein, ${ }^{6}$ sex, and age. The results of these 2 models allowed assessment of the magnitude of the direct effect of therapy not mediated through occurrence of nausea. Model fits were screened for the presence of outliers and for lack of fit using the Hosmer-Lemeshow test. Analyses were performed using Microsoft Excel, SPSS, or Stata v. 12.0 (College Station, TX).

RESULTS A total of 274 patients with CM underwent inpatient DHE treatment during the study period, of whom 153 were already on migraine preventives. Headache outcome information was available in 214 (78\%) patients. Seven of 274 patients did not achieve their target cumulative dose of $11.25 \mathrm{mg}$, since when nausea was present, antiemetic therapy was escalated.

Those with missing outcome data did not differ in terms of age, sex, or likelihood of having nausea or medication overuse. Overall, 78\% of patients with follow-up data (167/214) had medium-term headache benefit following DHE treatment. See table 1 for demographic and clinical information.
Table 1 Demographic and clinical data on 214 chronic migraine patients treated with IV dihydroergotamine (DHE)

\begin{tabular}{ll} 
Characteristics & Values \\
Age at treatment, y, mean \pm SD & $44 \pm 14$ \\
Age at treatment, y, median, min-max & 46 (16-80) \\
\% Female & 77 \\
$\begin{array}{l}\text { Headache exacerbation with DHE } \\
\text { infusion, \% }\end{array}$ & 21 \\
$\begin{array}{l}\text { Medium-term headache benefit, \% } \\
\text { Medication overuse, \% }\end{array}$ & 78 \\
Nausea, \% & 30 \\
\hline
\end{tabular}

Headache exacerbation. Nineteen percent $(n=41)$ had headache exacerbation with DHE infusions. Of these, $66 \%$ had medium-term headache benefit compared to $82 \%$ of those who did not have headache exacerbation $(p=0.07)$. In univariate logistic regression modeling, headache exacerbation with DHE infusion was associated with reduced odds of medium-term headache benefit (odds ratio [OR] $0.43,95 \%$ confidence interval [CI] $0.20-$ $0.91, p=0.03)$.

However, in multivariate logistic regression analysis adjusted for nausea, leg cramping, medication overuse, sex, and age, headache exacerbation with DHE was no longer an independent predictor of medium-term headache benefit (OR 0.65) (table 2). In this model, observations from 2 individuals were excluded as outliers due to large observed influence on estimated coefficients and associated inferences. The Hosmer-Lemeshow goodness-of-fit test for the final model was consistent with an acceptable fit to the data $(p=0.93)$.

Risk factors for DHE outcome. Of modifiable risk factors for headache outcome, nausea had the largest impact on the OR estimate, as the adjusted estimate for medium-term headache benefit following DHEassociated headache exacerbation changed slightly from the marginal estimate when nausea was left out of the model (OR 0.51, 95\% CI 0.22-1.13, $p=0.09$ ), but was similar to that seen in the fully adjusted model when leg cramping (OR 0.65, 95\% CI $0.28-1.51, p=0.31$ ) or medication overuse (OR $0.61,95 \%$ CI $0.26-1.40, p=0.24)$ were removed.

Nausea. Presence of nausea was significantly associated with headache exacerbation with DHE infusions (OR 11.4, 95\% CI 1.51-86.15, $p=0.02$ ) in a model adjusting for age, sex, medication overuse, and leg cramping. Presence of nausea was an independent predictor of lower likelihood of medium-term headache benefit in the fully adjusted model (OR 0.12, 95\% CI $0.02-1.00, p=0.05)$, as was the presence of 
Table 2 Multivariate logistic regression model for predictors of medium-term headache benefit in chronic migraine patients treated with a course of IV dihydroergotamine ( $\mathrm{n}=196$ with complete data)

$\begin{array}{llll}\text { Predictor } & \text { Odds ratio } & \begin{array}{l}95 \% \text { Confidence } \\ \text { interval }\end{array} & p \text { Value } \\ \text { Infusion-related headache exacerbation } & 0.65 & 0.28-1.51 & 0.31 \\ \text { Nausea } & 0.12 & 0.02-1.00 & 0.05 \\ \text { Age (per 10 years) } & 1.68 & 1.24-2.28 & 0.001 \\ \text { Female sex } & 0.44 & 0.16-1.28 & 0.13 \\ \text { Medication overuse prior to treatment } & 0.42 & 0.18-0.97 & 0.04 \\ \text { Leg cramping } & 1.46 & 0.62-3.48 & 0.39\end{array}$

medication overuse (OR 0.42, 95\% CI 0.18-0.97, $p=0.04)$. Older age was strongly associated with odds of headache benefit, with $68 \%$ increased odds of benefit with each decade increase in age (OR $1.68,95 \%$ CI 1.24-2.28, $p=0.001$ ). Post hoc visual inspection (figure) suggests patients in their teens and 20s were less likely to benefit.

DISCUSSION In this study, transient headache exacerbation occurred in $19 \%$ of $\mathrm{CM}$ patients treated with IV DHE. In a univariate logistic regression model, transient headache exacerbation with DHE infusions was associated with decreased odds of headache benefit. However, multivariate logistic regression analysis suggests this was mediated by the presence of nausea. The majority (66\%) of patients with headache exacerbation still had medium-term headache benefit. In a separate model, presence of nausea was strongly associated with the occurrence of headache exacerbation (OR 11.4, 95\% CI 1.51-86.15), suggesting that adequate nausea

Figure Proportion of chronic migraine patients experiencing medium-term headache benefit from IV dihydroergotamine (DHE) treatment

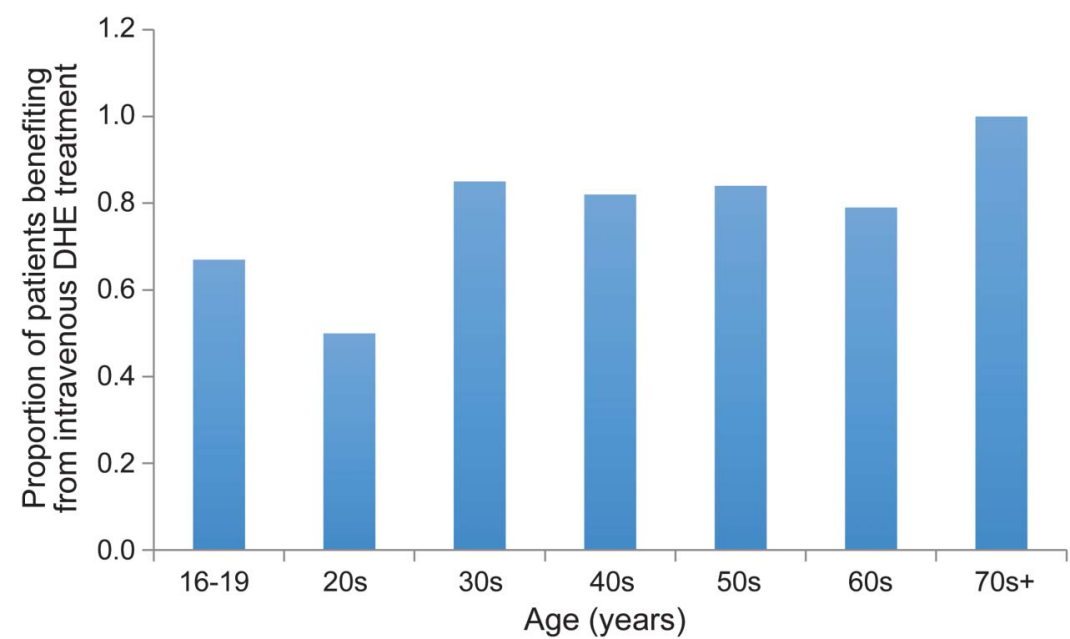

Histogram represents the likelihood of medium-term headache benefit from an inpatient course of DHE by age. control might help prevent such headache exacerbations.

The presence of transient headache exacerbation with DHE infusions has been reported previously.,7 It is not unique to DHE-headache exacerbation with triptans has been reported in adults ${ }^{8}$ and children. ${ }^{9}$ In this study, adequate nausea control was the most important modifiable factor predicting medium-term headache benefit with DHE infusions for CM. Experimental work demonstrates a link between activation of nociceptive trigeminal pathways emesis neurons in the caudal-medial nucleus of the tractus solitarius ${ }^{10}$ that may mediate this effect.

Younger patients with CM were found to be relatively less likely to benefit from an inpatient course of IV DHE. This age effect has also been demonstrated in acute migraine treatment wherein patients under age 34 years were less likely to benefit from triptan treatment. ${ }^{11}$ This highlights an even greater need for nausea control in that population.

The limitations of this study include the retrospective design. It is possible some exacerbations of headache with DHE went unreported. Future studies of patients undergoing DHE treatment for CM should systematically query for this symptom. Although we reviewed patients at 6 weeks and their preventive plan was almost invariably unchanged from discharge, the preventives initiated may have been a contributor to the overall outcome. This does not itself subtract from our observation concerning the worsening phenomenon. Our patient population was a tertiary headache clinic population, though we do not think this substantively limits the generalizability of these results for all patients undergoing a course of IV DHE treatment for CM. Clinicians in all settings should focus their efforts on aggressive nausea control during a course of IV DHE for chronic migraine.

\section{AUTHOR CONTRIBUTIONS}

Michael Eller: abstracted the data, drafted sections of the manuscript, and performed statistical analyses. Amy Gelfand: performed statistical analyses and drafted sections of the manuscript. Nina Riggins: assisted with data collection and revised the manuscript for intellectual content. Stephen Shiboski: assisted with statistical analyses and revised the manuscript for intellectual content. Cristoph Schankin: assisted with data collection and revised the manuscript for intellectual content. Peter J. Goadsby: assisted with data collection, supervised the study, and revised the manuscript for intellectual content.

\section{ACKNOWLEDGMENT}

The authors thank Janet Corroo, RN, Mei Mei Church, NP, Denise Chou, MD, Amy Tso, MD, Abraham Nagy, MD, and Teshame Monteith, MD, for patient care and documentation.

\section{STUDY FUNDING}

The King's CRF is funded by the NIHR and by the South London and the Maudsley Mental Health BRC. The views expressed are those of the authors and not necessarily those of the UK NHS, the NIHR, or the Department of Health, UK. Assistance with SPSS: CTSI grant UL1 TR000004. 


\section{Comment:}

\section{Outcomes of migraine therapy with IV dihydroergotamine}

IV dihydroergotamine (DHE) is a widely used treatment for status migrainosus or refractory chronic migraine, but there are few published studies on outcomes of this therapy. ${ }^{1}$ The report by Eller et al. ${ }^{2}$ adds to our current understanding of the effects of DHE, with a thorough description of their experience at an academic inpatient headache unit. Although the observations are retrospective, this kind of careful documentation and systematic reporting of patient experience can be enormously valuable in characterizing "real world" patient management issues. One such issue is the transient worsening of headache associated with migraine treatment, a phenomenon that occurs not only with DHE, but also not uncommonly with triptans. This intriguing response may have something to tell us about basic mechanisms of headache. From a practical standpoint, the data presented by Eller et al. tell us that transient worsening of headache with DHE does not predict a worse outcome, and is not a reason to discontinue therapy.

A second important issue is the identification of nausea as a risk factor for worse outcome with DHE treatment. Population studies have found that persistent frequent nausea is associated with increased risk of progression from episodic to chronic migraine. ${ }^{3}$ Whether or not treatment of nausea as a "modifiable risk factor" actually changes the clinical course of migraine remains unclear. What is clear, however, is that nausea is a frequently disabling component of migraine that warrants aggressive therapy. Despite a variety of therapeutic options for nausea, no evidence clearly supports one approach vs another. This study underscores the importance of optimizing therapy of migraine-related nausea, and of coming to a better understanding of the role of nausea and its treatment in the short- and long-term outcomes of patients with migraine.

1. Nagy AJ, Gandhi S, Bhola R, Goadsby PJ. Intravenous dihydroergotamine for inpatient management of refractory primary headaches. Neurology 2011;77:1827-1832.

2. Eller M, Gelfand AA, Riggins NY, Shiboski S, Schankin C, Goadsby PJ. Exacerbation of headache during dihydroergotamine for chronic migraine does not alter outcome. Neurology 2016;86:856-859.

3. Reed ML, Fanning KM, Serrano D, Buse DC, Lipton RB. Persistent frequent nausea is associated with progression to chronic migraine: AMPP study results. Headache 2015;55:76-87.

Andrew Charles, $M D$

From the David Geffen School of Medicine, UCLA, Los Angeles, CA.

Study funding: No targeted funding reported.

Disclosure: A. Charles has served on scientific advisory boards for Amgen, eNeura, and Eli Lilly; serves as an associate editor of Cephalalgia; and has received research support from Takeda Pharmaceuticals and the US Department of Defense. Go to Neurology.org for full disclosures.

\section{DISCLOSURE}

M. Eller reports no disclosures relevant to the manuscript. A. Gelfand receives grant support from NIH/NCATS (8KL2TR000143-09) and the Migraine Research Foundation, as well as honoraria for submissions from Journal Watch Neurology and personal compensation for legal consulting. N. Riggins, S. Shiboski, and C. Schankin report no disclosures relevant to the manuscript. P. Goadsby reports grants and personal fees from Allergan, grants and personal fees from eNeura, personal fees from Autonomic Technologies Inc., grants and personal fees from Amgen, personal fees from Bristol-Myers Squibb, personal fees from AlderBio, personal fees from Pfizer, personal fees from Zogenix, personal fees from Nevrocorp, personal fees from Impax, personal fees from DrReddy, personal fees from Zosano, personal fees from Colucid, personal fees from Eli-Lilly, personal fees from Medtronic, personal fees from Avanir, personal fees from Gore, personal fees from Ethicon, personal fees from Heptares, personal fees from Nupathe, personal fees from Ajinomoto, and personal fees from Teva, outside the submitted work. Go to Neurology.org for full disclosures.

Received January 12, 2015. Accepted in final form September 8, 2015.

\section{REFERENCES}

1. Lipton RB. Chronic migraine, classification, differential diagnosis, and epidemiology. Headache 2011;51(suppl 2):77-83.

2. Raskin NH. Repetitive intravenous dihydroergotamine as therapy for intractable migraine. Neurology 1986; 36:995-997.

3. Nagy AJ, Gandhi S, Bhola R, Goadsby PJ. Intravenous dihydroergotamine (DHE) for inpatient management of refractory primary headaches. Neurology 2011;77: 1827-1832.

4. Weatherall MW, Telzerow AJ, Cittadini E, Kaube H, Goadsby PJ. Intravenous aspirin (lysine acetylsalicylate) in the in-patient management of headache. Neurology 2010;75:1098-1103.

5. Headache Classification Committee of the International Headache Society. The International Classification of Headache Disorders, 3rd edition (beta version). Cephalalgia 2013;33:629-808.

6. Perren MJ, Feniuk W, Humphrey PPA. Vascular 5-HT1like receptors that mediate contraction of the dog isolated saphenous vein and carotid arterial vasoconstriction in anaesthetised dogs are not of the 5-HT1A or 5-HT1D subtype. Br J Pharmacol 1991;102:191-197.

7. Callaham M, Raskin N. A controlled study of dihydroergotamine in the treatment of acute migraine headache. Headache 1986;26:168-171.

8. Dahlof CG. How does sumatriptan perform in clinical practice? Cephalalgia 1995;15:21-28.

9. Linder SL. Subcutaneous sumatriptan in the clinical setting: the first 50 consecutive patients with acute migraine in a pediatric neurology office practice. Headache 1996; 36:419-422.

10. Kaube H, Keay KA, Hoskin KL, Bandler R, Goadsby PJ. Expression of c-Fos-like immunoreactivity in the caudal medulla and upper cervical cord following stimulation of the superior sagittal sinus in the cat. Brain Res 1993;629: 95-102.

11. Diener HC, Ferrari M, Mansbach H. Predicting the response to sumatriptan: the sumatriptan naratriptan aggregate patient database. Neurology 2004;63:520-524.

\section{Get Connected. Stay Connected.}

Connect with the American Academy of Neurology's popular social media channels to stay up-todate on the latest news and breakthroughs in neurology, and network with peers and neurology thought leaders. Visit AAN.com/Connect. 